\title{
Docencia en tiempos de distanciamiento social
}

\section{Teaching in times of social distancing}

\author{
Mariela Torrente A. ${ }^{1}$
}

Un puñado de cuadrados negros con nombres de personas y símbolos de micrófonos apagados. ¿Realmente habrá alguien escuchando? Me recomiendan que haga preguntas de vez en cuándo, y si nadie contesta? Sería la evidencia de que realmente a nadie le importa lo que estoy diciendo, situación probable.

La emergencia sanitaria derivada del COVID-19 ha afectado nuestras vidas de muchas maneras, tanto en el plano personal como laboral. La actividad docente no podía estar ajena a este tsunami. A lo largo de nuestras carreras hemos recibido consejos de cómo realizar presentaciones efectivas, tanto en clases de pre y postgrado como en reuniones científicas. El objetivo final es cautivar al espectador/a y transmitir un mensaje claro. Esto se lograba con una mezcla de apoyo audiovisual y carisma. En los últimos meses esto cambió.

Una de las consecuencias de la cuarentena ha sido la migración obligada de docencia presencial a docencia remota. La metodología de e-learning, o aprendizaje basado en recursos computacionales $(\mathrm{ABC})$, ha tenido un sostenido desarrollo en los últimos 10 años, incluidas las profesiones del área de la salud. ABC es al menos tan efectivo como el aprendizaje presencial $^{1,2}$. Lo mismo ocurre en el área de Otorrinolaringología, tanto para el pregrado como para el postgrado ${ }^{3}$.

Los recursos docentes que se utilizan en $\mathrm{ABC}$ son diversos y tienen como objetivo desarrollar la capacidad de razonamiento clínico ${ }^{4}$. Por ejemplo, se puede recurrir a casos clínicos con árbol de decisiones donde el/la alumno/a vaya progresando en la medida que elige la decisión correcta (simulando un juego). En este caso es importante el feedback en cada toma de decisión, tanto para la decisión correcta como incorrecta, lo cual se puede realizar con un "avatar" que chatee online con mensajes previamente grabados, con asesorías en tiempo real o con foro de discusión. En las especialidades quirúrgicas es posible desarrollar tutoriales de técnicas para procedimientos específicos, ya sea con videos de casos reales o nuevamente con técnica de árbol de decisiones. Las posibilidades son múltiples.

Ahora bien, la pandemia no dio tiempo para una adecuada preparación en competencias de educación a distancia, tradicionalmente la docencia en carreras de la salud en la mayoría de los países es presencial. Entre las barreras para el desarrollo del $\mathrm{ABC}$ están los costos, falta de desarrollo de softwares adecuados, falta de competencias en el recurso humano $y$ una actitud negativa hacia el tema por parte de alumnos/as y docentes 5 . Los organismos relacionados con la educación en salud, universidades y sociedades científicas, tienen un gran desafío en este campo. E-learning puede ser una oportunidad en nuestra especialidad ya que permite el acceso a más personas, ejemplo de esto es la masiva asistencia a actividades online (webinars) en comparación con actividades científicas presenciales.

Es así como la educación a distancia debería ser una nueva herramienta en la formación de Otorrinolaringología y educación continua, no cuestionarnos si debemos usarla, sino cuándo y cómo ${ }^{6}$, y desarrollar las competencias necesarias para ello. La emergencia sanitaria ha tenido muchos efectos negativos, tanto a nivel personal como social, pero también puede ser fuente de nuevas oportunidades.

\section{Bibliografía}

1. Vaona A, Banzi R, Kwag KH, et al. E-learning for health professionals. Cochrane Database Syst Rev 2018;1:CD011736. 


\section{EDITORIAL}

2. Maertens H, Madani A, Landry T, Vermassen F, Van Herzeele I, Aggarwal R. Systematic review of e-learning for surgical training. Br J Surg 2016;103:1428-1437.

3. Tarpada SP, Hsueh WD, Gibber MJ. Resident and student education in otolaryngology: A 10year update on e-learning. Laryngoscope 2017; 127:E219-E224.

4. Deschênes MF, Goudreau J, Fontaine G, et al. Theoretical foundations of educational strategies used in e-learning environments for developing clinical reasoning in nursing students: A scoping review. Nurse Educ Pract 2019;41:102632.

5. Childs S, Blenkinsopp E, Hall A, Walton G. Effective e-learning for health professionals and studentsbarriers and their solutions. A systematic review of the literature-findings from the HeXL project. Health Info Libr J 2005;22:20-32.

6. Cook DA, Garside S, Levinson AJ, Dupras DM, Montori VM. What do we mean by web-based learning? A systematic review of the variability of interventions. Med Educ 2010;44:765-774. 